\title{
Spectrum delivery function in distributed antenna system using Radio on Free Space Optics for indoor WLAN
}

\author{
Misato Kamei ${ }^{a)}$, Takeshi Higashino, Katsutoshi Tsukamoto, \\ and Shozo Komaki \\ Division of Electrical, Electronic and Information Engineering, \\ Graduate School of Engineering, Osaka University \\ 2-1 Yamada-oka, Suita-shi, Osaka, 565-0871, Japan \\ a)kamei@roms.comm.eng.osaka-u.ac.jp
}

\begin{abstract}
This letter proposes a spectrum delivery function in distributed antenna system (DAS) using Radio on Free Space Optics (RoFSO) for indoor wireless local area network (WLAN). RoFSO can transfer various kind of radio services on optical wireless carrier, and spectrum delivery function realizes efficient utilization of radio frequency (RF) resources. Numerical calculations show that the improvement in throughput performance can be obtained when users are locally concentrated, and RF resources are fairly used in terms of fairness index.
\end{abstract}

Keywords: Radio on Free Space Optics (RoFSO), distributed antenna system, indoor WLAN, spectrum delivery function

Classification: Microwave and millimeter wave devices, circuits, and systems

\section{References}

[1] K. Tsukamoto, "Radio on Fiber (RoF) Technology and It's Applications," IEICE Technical Report, OCS2002, vol. 102, no. 358, pp. 22-28, Oct. 2002.

[2] H. H. Refai, J. J. Sluss, Jr., H. H. Refai, and M. Atiquzzaman, "Transporting RF signals over Free-Space Optical Links," Proc. of the SPIE, vol. 5712, issue 1, pp. 46-54, 2005.

[3] H. Harada, S. Kajiya, K. Tsukamoto, S. Komaki, and N. Morinaga, "TDM Intercell Connection Fiber-Optic Bus Link for Personal Radio Communication System," IEICE Trans. Commun., vol. E78-B, no. 9, pp. 1287-1294, Sept. 1995.

[4] M. Morikura and S. Kubota, 802.11 High-Speed Wireless LAN Textbook, impress, Tokyo, 2005.

[5] M. Kamei, A. Murakoshi, T. Higashino, K. Tsukamoto, and S. Komaki, "Proposal of Distributed Antenna System Using Radio on Free Space Optics for Indoor WLAN and Its Throughput Improvement with Spectrum Delivery Function," Proc. AP-MWP 2006, Kobe, Japan, H-4, pp. 285-288, April 2006. 
[6] D. M. Chiu and R. Jain, "Analysis of the increase and decrease algorithms for congestion avoidance in computer networks," J. Comput. Networks ISDN Syst., vol. 17, pp. 1-14, June 1989.

\section{Introduction}

Recently, various types of mobile terminals (MTs) and various wireless access system have been widely used at outdoor, office, home, factory and so on. A serious problem is the lack of frequency resources that causes poor performance of wireless services. In order to satisfy users' demands for high speed and high quality transmission, efficient utilization of radio frequency (RF) resources is strongly required. For example, in wireless local area network (WLAN) system, multiple access points (APs) are likely to be available to accommodate a lot of users. However, throughput performance in WLAN rapidly degrades when a lot of users access to a particular AP due to their random access and statical allocation of RF channels. Then, unfair throughput among mobile users is provided. Therefore, to mitigate a degradation of throughput performance, flexible allocation of RF resource is required.

Radio-on-Fiber (RoF) network is a candidate of an universal network for providing various wireless services. In micro-cellular system, RoF technique realizes efficient utilization of $\mathrm{RF}$ resources with simple equipment of radio base stations (RBSs) with only function of E/O (Electrical-to-Optical) and $\mathrm{O} / \mathrm{E}$ (Optical-to-Electrical) conversions [1]. RoF link connects RBSs and a central control station (CCS). RF signals are transmitted over RoF link in which RF signals keep their radio format. The CCS has the functions of modulation and demodulation and channel allocation. Then, flexible allocation of RF resources becomes possible. For indoor use, however, the fiber construction needs a large investment, and once fiber cables has been laid, re-wiring is difficult even if the distribution of MTs and the number of MTs are changed.

Radio on Free Space Optics (RoFSO) technique $[1,2]$ transfers RF signals on an optical wireless carrier. Therefore, the RoFSO network has advantage of both the RoF network and the wireless network. In this letter, a spectrum delivery function in distributed antenna system (DAS) using RoFSO is proposed. Proposed function realizes efficient frequency utilization in terms of both throughput performance and fairness index.

\section{RF spectrum delivery function for DAS using RoFSO}

Figure 1 (a) illustrates the system configuration of DAS using RoFSO. This system is composed of SDRGW (Software Definable Radio Gateway) with CCS and RBSs. SDRGW seamlessly connects IP network to RoFSO network. First, SDRGW converts IP packets into RF signals. At the CCS, each RF signals are allocated to LD (Laser Diode) corresponding to the destination by controlling the spectrum delivery switch. Then the RF signals modulate 


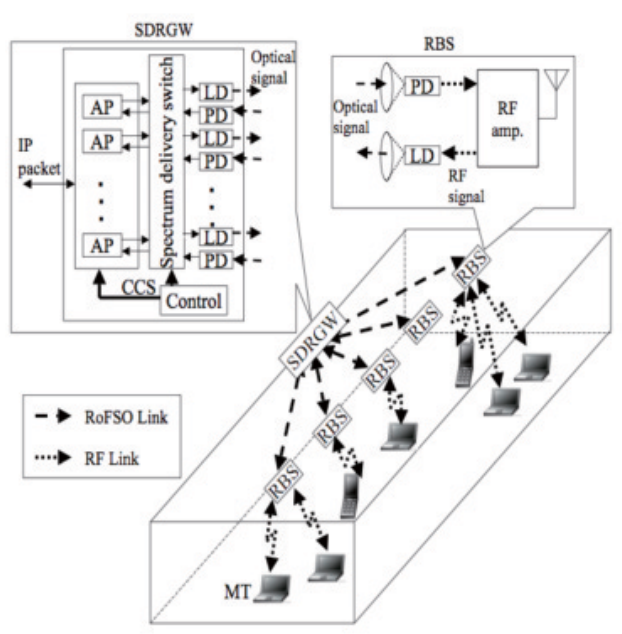

(a)

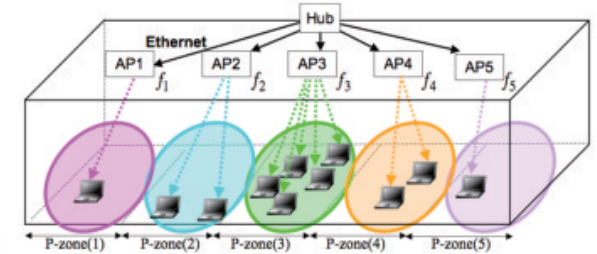

(b)

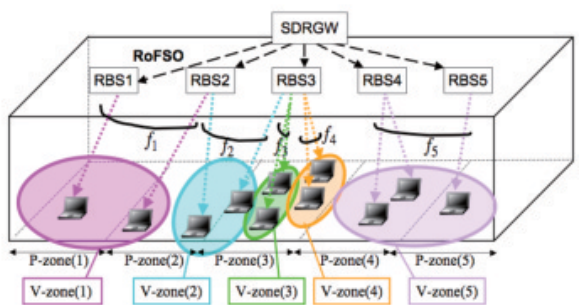

(c)

Fig. 1. (a) System configuration of the proposed DAS using RoFSO and spectrum delivery function: (b) conventional system and (c) proposed system

LDs with intensity modulation (IM) format. The IM signal is transmitted to the RBS through free space optical link. At the RBS, the received IM signal is converted to the RF signals by PD (Photo Diode). The RF signals are transmitted to an appropriate MT. Figure 1(b) and (c) illustrate comparison of conventional system and proposed system in indoor WLAN. In the conventional system, APs are connected each other with Ethernet. Each AP uses one RF channel and covers one physical zone (P-zone). MTs select one AP based on strength of received RF signal power. Therefore, MTs are likely to select the nearest AP. That is, MTs belong to the P-zone which the nearest AP covers. The basic access control of the IEEE 802.11 MAC (Media Access Control) protocol is called CSMA/CA (Carrier Sense Multiple Access with Collision Avoidance) and this protocol defines an optional four-way handshaking technique called RTS/CTS (Request to Send/Clear to Send) to combat the hidden terminal problems. MTs belonging to the same P-zone share one RF channel by using CSMA/CA with RTS/CTS.

On the other hand, in the proposed system, CCS can allocate several RF channels to RBS with the spectrum delivery switch and MTs share each channel over several P-zones. That is, the proposed system configures virtual zones (V-zones) whose sizes are freely variable, and it makes each RF channel shared by the same number of MTs.

In the proposed system, we consider the situation in which LD correspond one-to-one with RBS (Proposed1). Proposed system has advantage of being able to provide many RF channels to the RBS which covers the area where MTs are concentrated. However, when one optical carrier transports many RF channels as subcarrier, reduction of modulation index causes degradation in throughput performance. Therefore, we additionally propose introduction of space-division multiplexing (SDM) in which multiple optical wireless carriers transport RF signals in parallel in order to prevent a loss of optical modulation index (Proposed2). Because RoFSO links are wireless, it is eas- 
ily possible to allocate multiple LDs to one RBS depending on locations of MTs. We consider the situation in which two LDs are allocated to the RBS that cover the largest number of MTs and evaluate this effect in the following section.

\section{Numerical calculation models}

Performance of the proposed system is evaluated by numerical calculation. We assume the service area in a room with $60 \mathrm{~m}$ in width, $20 \mathrm{~m}$ in depth and $3 \mathrm{~m}$ in height as shown in Fig. 2 (a). The service area is divided into P-zones of the same number as RBSs or APs. RBSs or APs are located at the center of each P-zone on the ceiling (RBSs or APs are placed evenly spaced apart on red line in Fig. 2 (a)). The number of RF channels used in this service area is assumed to be the same number of RBSs or APs. RF signals compliant with IEEE Standard 802.11a (Figure 2 (a) shows the situation in 3 channels (3 RBSs or 3 APs are located and 3 channels are used in service area)). It is assumed that MTs are distributed on uniform distribution in depth direction and Gaussian distribution in width direction that centers on $30 \mathrm{~m}$ point.

We have previously analyzed the propagation property of the proposed system in [5] and have derived received CNR (Carrier-to-Noise Power Ratio) at an MT. We assume that the transmission mode in WLAN (the adaptive modulation: BPSK, QPSK, 16QAM and 64QAM, the coding rate: 1/2, 2/3, $3 / 4$ ) is set according to received CNR at an MT.

In the conventional system, transmission $\mathrm{RF}$ power radiated from an $\mathrm{AP}$ is assumed to be $5 \mathrm{~mW}$. In the proposed system, RF carrier power after PD detection, $P_{R F-R B S}$, is represented as [3]

$$
P_{R F-R B S}=\frac{1}{2} m_{s}^{2} \alpha^{2} P_{r-o p t}^{2},
$$

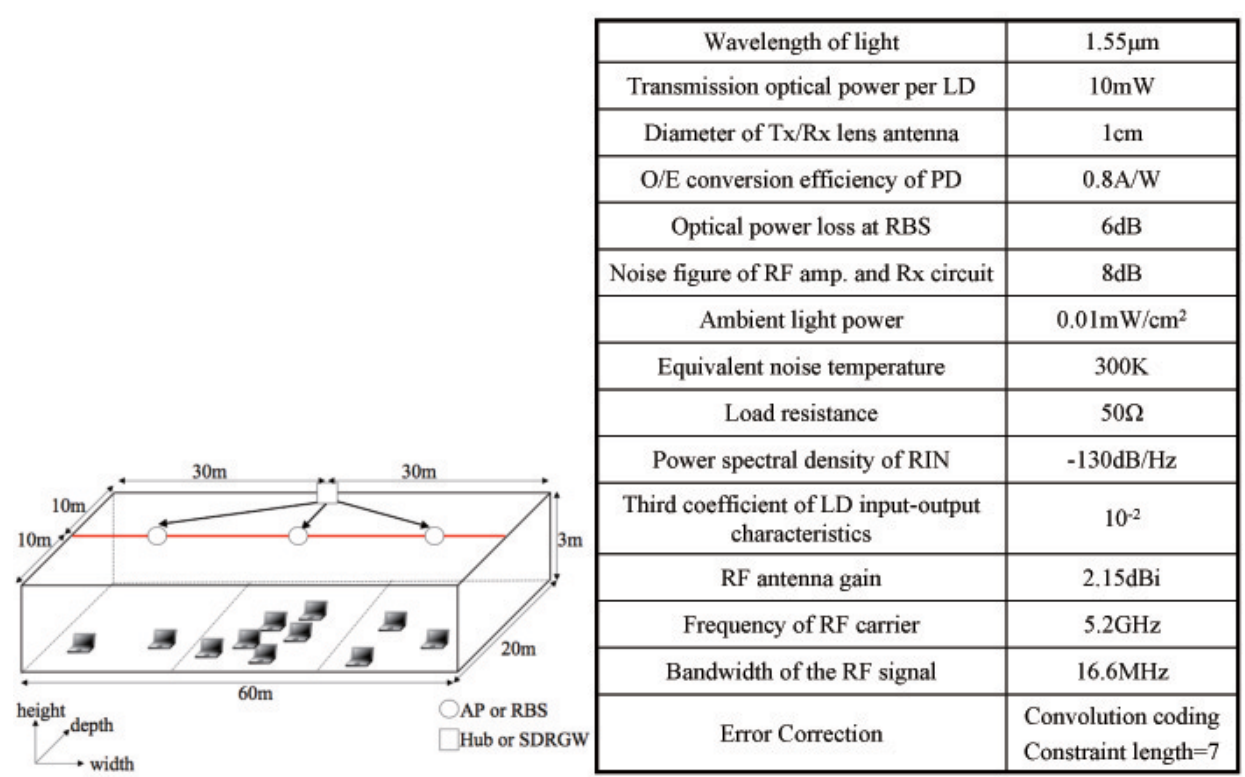

(a) (b) 
where $m_{s}$ is the optical modulation index for $s$-th MT, $\alpha$ is the O/E conversion efficiency of $\mathrm{PD}$, and $P_{r-o p t}$ is received optical power at $\mathrm{PD}$. After $\mathrm{PD}$ detection, $\mathrm{RF}$ power is amplified so that $\mathrm{RF}$ power radiated from a RBS becomes $5 \mathrm{~mW}$ when $m_{s}=1$. To prevent the over-modulation, $m_{s}$ is set to the inverse of the number of RF channels that are transported on an optical wireless carrier.

The propagation loss between a RBS or an $\mathrm{AP}$ and an MT, $L_{R F}[\mathrm{~dB}]$, is given by [4],

$$
L_{R F}=20 \log _{10} f+K \log _{10} r-28,
$$

where $f[\mathrm{MHz}]$ is the frequency of $\mathrm{RF}$ carrier, $K$ is distance attenuation coefficient (In the indoor environment such as office room, $K$ for $5.2 \mathrm{GHz}$ band is 31), and $r$ is distance between a RBS or an AP and an MT.

We assume that error of the control packets doesn't occur. In situations that $M$ MTs share one RF channel, the throughput per an MT, $\tau$, is

$$
\tau=\frac{\text { Data } \cdot\left(1-P_{P E R}\right)}{M \cdot t_{T}},
$$

where $t_{T}$ is the mean time required to be sent and received of a data frame including the back-off time, the time for Inter frame spaces (IFS) and the time for transmission of data, RTS, CTS and ACK (ACKnowledgement). $P_{P E R}$ is packet error rate and Data is the data flame length (We assume 1000 octet).

Fairness index, $F$, is given by $[6]$,

$$
F=\frac{\left\{\sum_{i=1}^{M_{\text {total }}} \tau_{i}\right\}^{2}}{M_{\text {total }} \cdot\left\{\sum_{i=1}^{M_{\text {total }}} \tau_{i}^{2}\right\}}
$$

where $M_{\text {total }}$ is total number of MTs in service area and $\tau_{i}$ is throughput of $i$-th MT. Parameters used in numerical calculation are shown in Fig. 2 (b).

\section{Numerical results}

Figure 3 (a) shows standard deviation of Gaussian distribution versus throughput per an MT when $M_{\text {total }}=30$. Small standard deviation means the situa-

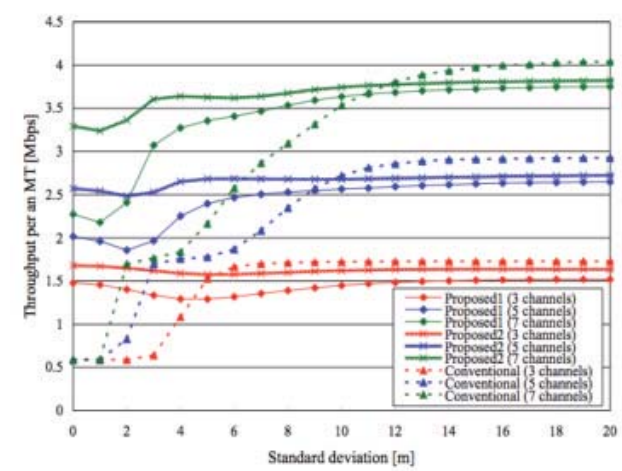

(a)

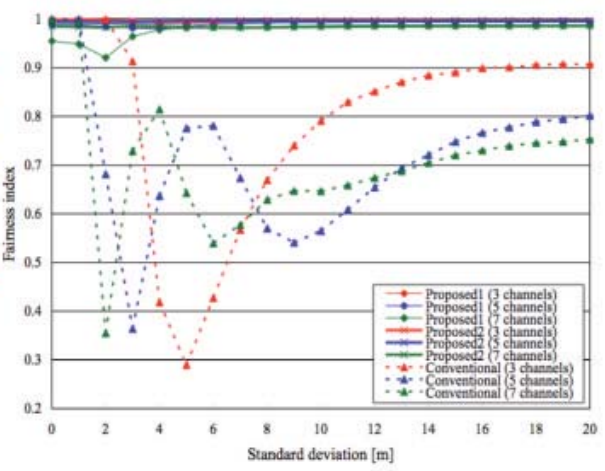

(b)

Fig. 3. Standard deviation of Gaussian distribution versus (a) Throughput per an MT and (b) Fairness index 
tion in which MTs are locally concentrated. In the conventional system, rapid degradation of throughput is seen when standard deviation is small. When the number of RF channels is increased, the degradation is larger (When 7 channels are used and standard deviation is from $20 \mathrm{~m}$ to $0 \mathrm{~m}$, throughput per an MT is degraded by 3.5 Mbps). In the Poposed1, the degree of degradation is smaller than the conventional system. However, when the number of $\mathrm{RF}$ channels are increased, the degree of degradation increases in the situation that MTs are locally concentrated, because many RF channels are transported on one optical wireless carrier and the transmission RF power per one RF channel at a RBS becomes small due to a loss of optical modulation index. Proposed2 improves throughput performance compared with proposed1. When 7 channels are used, throughput per an MT is improved about 1.0 Mbps only by adding one optical wireless carrier allocated to the RBS that cover the largest number of MTs. When standard deviation are increase (MTs become to be uniformly distributed), throughput per an MT in the proposed system is smaller than the conventional system due to a loss of optical modulation index.

Figure 3 (b) shows standard deviation of Gaussian distribution versus fairness index when $M_{\text {total }}=30$. In the proposed system, fairness index is almost always 1 that indicates better fairness among MTs. In the conventional system, it is seen that unfairness of throughput among MTs is caused. When MTs are locally concentrated, fairness index is 1 because all MTs have fairly poor throughput. As standard deviation increases, fairness index comes to decrease because some MTs begin to connect next APs on which MTs are not concentrated. Then, when standard deviation increases further, fairness index comes to increase because the number of MTs which connect next APs increases. When MTs become to be uniformly distributed, fairness index approach 1. However, unfairness of throughput among MTs remains. The proposed system can realize that MTs use RF resources fairly and solve the problem of unfairness in the conventional system.

\section{Conclusion}

This letter has proposed a spectrum delivery function in DAS using RoFSO for indoor WLAN. Numerical calculations show that the improvement in throughput can be obtained when MTs are locally concentrated, and fairness index is almost always 1 in the proposed system even if standard deviation of MTs' distribution is changed. MTs can use RF resources fairly and efficiently in the proposed system even if MTs are locally concentrated.

\section{Acknowledgments}

This letter is partially supported by the Grants-in-Aid for Exploratory Research No. 17656125, from the Japan Society for the Promotion of Science. 